\title{
Bulk Enthalpy Calculations in the Arc Jet Facility at NASA ARC
}

\author{
Corinna S. Thompson ${ }^{*}$ \\ Sandia National Laboratories, Albuquerque, NM, 87123 \\ Dinesh Prabhu ${ }^{\dagger}$ \\ ERC Inc., NASA Ames Research Center, Moffett Field, CA, 94035 \\ Imelda Terrazas-Salinas ${ }^{\ddagger}$ \\ NASA Ames Research Center, Moffett Field, CA, 94035 \\ and \\ Jeffrey J. Mach ${ }^{\S}$ \\ Sierra Lobo Inc., NASA Ames Research Center, Moffett Field, CA, 94035
}

The Arc Jet Facilities at NASA Ames Research Center generate test streams with enthalpies ranging from $5 \mathrm{MJ} / \mathrm{kg}$ to $25 \mathrm{MJ} / \mathrm{kg}$. The present work describes a rigorous method, based on equilibrium thermodynamics, for calculating the bulk enthalpy of the flow produced in two of these facilities. The motivation for this work is to determine a dimensionally-correct formula for calculating the bulk enthalpy that is at least as accurate as the conventional formulas that are currently used. Unlike previous methods, the new method accounts for the amount of argon that is present in the flow. Comparisons are made with bulk enthalpies computed from an energy balance method. An analysis of primary facility operating parameters and their associated uncertainties is presented in order to further validate the enthalpy calculations reported herein.

\section{Nomenclature}

$\begin{array}{ll}A^{*} & =\text { throat area of sonic nozzle }\left(\mathrm{m}^{2}\right) \\ u & =\text { velocity }(\mathrm{m} / \mathrm{s}) \\ a^{*} & =\text { sonic velocity at the nozzle throat }(\mathrm{m} / \mathrm{s}) \\ \mathrm{c} & =\text { curve-fit constant } \\ H_{o} & =\text { stagnation state enthalpy }(\mathrm{J} / \mathrm{kg}) \\ h_{o 1} & =\text { initial guess for stagnation state enthalpy }(\mathrm{J} / \mathrm{kg}) \\ P_{o} & =\text { stagnation state pressure }(\mathrm{Pa}) \\ p^{*} & =\text { pressure at the nozzle throat }(\mathrm{Pa}) \\ \sigma & =\text { sonic flow parameter }(\mathrm{s} / \mathrm{m}) \\ S_{o 1} & =\text { initial guess for stagnation state entropy }(\mathrm{J} / \mathrm{kg} . \mathrm{K}) \\ T_{o 1} & =\text { initial guess for stagnation state temperature }(\mathrm{K}) \\ w & =\text { mass flow rate }(\mathrm{kg} / \mathrm{s}) \\ x & =\text { curve-fit exponent } \\ \gamma & =\text { isentropic exponent }\end{array}$

\footnotetext{
${ }^{*}$ Mechanical Engineer, Firing Sets and Impact Fusing, MS 0344, AIAA Member

${ }^{\dagger}$ Senior Research Scientist, Aerothermodynamics Branch, MS 230-3, Associate Fellow AIAA

‡ Test Engineering Lead, Thermophysics Facilities Branch, MS 229-2

${ }^{\S}$ Data Systems Group Supervisor, Thermophysics Facilities Branch, MS 229-2
} 


\section{Introduction}

A $\mathrm{n}$ important quantity required for computation of the thermal response of a material exposed to arc-heated flow, and also for modeling and simulation of the flow of an arc-heated gas mixture through a convergent-divergent nozzle, is the energy content (thermal, chemical, and kinetic energy), or simply the enthalpy of the flow. ${ }^{1}$ An estimate of this quantity is reported along with other parameters such as the arc current and voltage, the mass flow rates of all gases (primarily air and argon), the pressure developed in the arc column, etc., as part of the standard practice of arc jet testing at NASA Ames Research Center (ARC). Traditionally, theoretical estimates of the bulk enthalpy of the flow for various arc-heater settings are reported. These are often supplemented with values deduced from measurement of the temperature rise of water used in cooling the facility, in a method known as enthalpy by energy balance ${ }^{2}$ (EB2). There is not always agreement between these various estimates, and a single consistent estimate of the bulk enthalpy is desirable for a given arc-heater setting.

We note that the real quantity of interest in arc jet testing, especially in stagnation mode, is the enthalpy at the centerline of the nozzle. For the cases where the core flow is uniform, the distinction between bulk enthalpy and centerline enthalpy may be too fine to make. However, the core flow could be non-uniform, in which case, distinction needs to be made between the bulk and centerline enthalpies. The present work focuses on the bulk enthalpy of the flow slug delivered to the plenum by the arc heater, and not on the thermochemical processes involved in the expansion of the arc-heated slug of gas through the convergent-divergent nozzle.

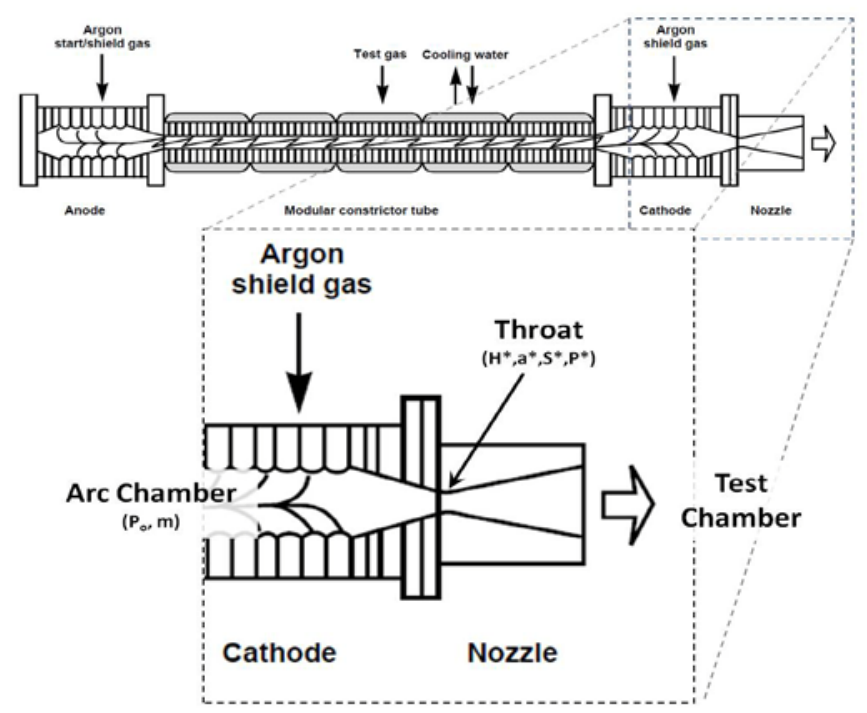

Figure 1: Schematic diagram of the segmented arc heaters used at Ames Research Center. ${ }^{3}$

Two of the "traditional" methods to determine bulk enthalpy of the arc jets at NASA ARC are described in this section and are used as the basis for an improved calculation. A third method, the EB2 method, which is independent and complimentary, will be used for comparison in subsequent sections.

The standard practice in arc jet testing at NASA ARC is to report bulk enthalpies calculated using the Winovich ${ }^{4}$ empirical formula (Eqs. 1). (The correlation of Winovich is assumed valid over the enthalpy range between 1000 and 10,000 Btu/lbm (equivalently 2.3 to $23 \mathrm{MJ} / \mathrm{kg}$ ).)

$$
w / A^{*} P_{o}=280 / H_{o}^{0.397}
$$

Rearranging the empirical formula for enthalpy,

$$
H_{o}=\left(280 /\left[w / A^{*} P_{o}\right]\right)^{2.52}=(280 / \sigma)^{2.52}
$$

where the sonic flow parameter, $\sigma$, is defined in equation 3 and has units of $\mathrm{kg} / \mathrm{sec} \mathrm{m}^{2} \mathrm{~atm}$. 


$$
\sigma=w / A^{*} P_{o}
$$

The SI equivalent of Eq. (2) is ${ }^{4}$ :

$$
H_{o}=(123 / \sigma)^{2.52}
$$

We note that enthalpy has units of energy per unit mass, $\mathrm{m}^{2} / \mathrm{s}^{2}$ (equivalently $\mathrm{J} / \mathrm{kg}$ ), and the units of the sonic flow parameter are s/m. Requiring Eq. (4) to be dimensionally balanced means that the numerical constant must also have units raised to the 2.52 power which implies an ambiguous physical significance or modulus. Alternatively, the exponent in Winovich's empirical formula could be made 2 instead of 2.52, which would necessarily change the constant, but would keep with an equation proportional to the square of a velocity term.

A closer approximation, from a dimensional perspective, for bulk enthalpy is that of Shepard et. al. ${ }^{5}$ (Eq. 5). The exponent in this formula is much closer to 2, but the expression is still dimensionally incorrect.

$$
H_{o}=(158.7 / \sigma)^{1.971}
$$

Winovich, having done his work in the 1960s, used a combination of experimental reference data and Mollier diagrams ${ }^{6}$ for air to determine supersonic arc jet nozzle flow properties. Mollier diagrams are enthalpy-entropy plots ( $h$-s diagrams) showing lines of constant temperature, pressure, and density. The procedure that Winovich followed for his analysis is outlined by Yoshikawa and Katzen. ${ }^{7}$ In this methodology, the ratio of static to stagnation pressures are used in the determination of the equilibrium properties of the flow from a nozzle. The assumptions include thermal equilibrium, and a steady-state, one-dimensional, isentropic, homogeneous flow field.

Shepard et. al. studied sonic flow enthalpy in the early 1990s. Instead of using Mollier diagrams, they used an inhouse computer program, ARCFLO ${ }^{8}$, to predict airflow properties through a choked nozzle. ARCFLO calculated flow properties in the arc heater. The previously-stated flow assumptions were used, with the exception that the flow was treated as two-dimensional and axisymetric.

In both the Winovich and Shepard approaches, the idea was the same: determine theoretical values of bulk enthalpy over a range of sonic flow parameters, using either Mollier diagrams or computational thermodynamic codes, and derive an equation using those values assuming a power-law fit of the data (Eq. 6). The constant, $c$, and the exponent, $x$, were determined by fitting experimental data that the authors had at their disposal.

$$
H_{o}=(c / \sigma)^{x}
$$

In light of this brief background, the objectives of the present work are: (1) to develop a rigorous, dimensionally correct procedure for computing bulk enthalpy in arc jet flows that is dimensionally correct and determine whether or not the results from the improved procedure are in better agreement with experimental data from two of the arc jet facilities at ARC - the Aerodynamic Heating Facility (AHF) and the Interaction Heating Facility (IHF); (2) to evaluate the dependence of bulk enthalpy on the amount of argon shield gas; and (3) to develop an analysis procedure for determining uncertainties in the measurements. By examining the uncertainty in the different enthalpy calculations, conclusions will be drawn about their statistical similarity or dissimilarity.

\section{Method}

The goal of this work is the same as that of the previous efforts, but with the exception that the exponent, $x$, in Eq.(6) is fixed at a value of 2 for dimensional correctness. The thermodynamic code that supplies theoretical values is Chemical Equilibrium with Applications ${ }^{9}$ (CEA), also known as the Gordon-McBride code. CEA, unlike the two tools mentioned already, generates thermodynamic properties for various gas mixtures rather than just air alone. Furthermore, the operating pressures in the arc heater are usually on the order of 1 bar or greater, and the assumption of equilibrium flow is reasonable. We note that the arc jet facilities do operate at pressures less than 1 bar; however these tests were not considered in the present work.

The arc jets at NASA Ames Research Center use argon as a shield gas. Argon is injected into the flow to reduce erosion at the surface of the electrodes. It is also used as a mechanism to prevent intersegment arcing at the anode and cathode sections of the arc jet. ${ }^{3}$ CEA produces thermodynamic properties for the test specific argon-air mixture. 
There exists currently a large amount of heat flux data from calorimeter measurements performed in the AHF and the IHF for our analysis- much larger than the data sets that were at the disposal of Winovich or Shepard. For our analysis, the mass flow rate of air and argon and the arc-column pressure are taken from the facility database. The nozzle throat areas of the two facilities are known constants. Thus, a sonic flow parameter can be calculated for each test recorded in the database according to Eq. (3), and thus help in correlating the total enthalpy $H_{0}$ to $\sigma$ via Eq. (6).

A Matlab ${ }^{10}$ program that follows the iterative procedure suggested by Hightower ${ }^{11}$ was developed to determine enthalpy. This program accesses extensive, but discrete, arrays of outputs (enthalpy, entropy, free energy, isentropic exponent, etc.) previously generated from the CEA program, with argon content ranging from 0 to 50 percent in increments of 2.5 percent, stagnation state pressure varying from 0.1 to 10.8 bars in increments of 0.1 bar, and temperature varying from 250 to $9750 \mathrm{~K}$ in increments of $50 \mathrm{~K}$. An example of the information that is output by CEA is shown in Table (1). The stagnation state pressure, $P_{o}$, measured during an experiment, recorded in the facility database and reported as the arc-column pressure, is assumed to be accurate. The stagnation temperature is not known. At this point, the user has an array of thermodynamic property data to navigate. The stagnation state pressure is rounded to the nearest increment (the nearest $0.25 \mathrm{bar}$ ) and the file containing temperature-dependent data at that pressure is found. The file contains the information similar to that in Table (1) in text format. Because the stagnation state temperature is unknown, an initial enthalpy guess is made, $h_{o}$. The initial guess of enthalpy is a recorded enthalpy value from the Arc Jet database, derived using the method of either Shepard or Winovich, and discussed further in the results section. Two parameters have now been fixed. That initial enthalpy guess can then be queried from within the discreet array of temperature-dependent data. The temperature, entropy, and all of the other thermodynamic properties follow from the initial guess of enthalpy and knowledge of pressure: $T_{o}$, $S_{o}$, etc.

Properties of specific interest are the enthalpy, entropy, sonic velocity $\left(a^{*}\right)$, and the isentropic exponent $(\gamma)$. The use of the CEA program allows us to determine an isentropic exponent specific to the flow composition and in these cases it is not the 1.4 value for an ideal diatomic gas. The reader is directed to Table (1) where the thermodynamic properties are shown to vary by temperature for constant pressure, but at a specific composition. Given a set of stagnation state parameters, $P_{o}, h_{o}, T_{o}, S_{o}$, the thermodynamic properties at other locations in the supersonic nozzle configuration can be calculated, if the velocity of the flow is known, starting with Eq. (7). The sonic throat state can be found by calculating the pressure at the throat for Mach number equal to one and holding the entropy constant according to Eq. (8).

$$
p=P_{o}\left(1+[(\gamma-1) / 2] M^{2}\right)^{\gamma / 1-\gamma}
$$

At the throat, $\mathrm{M}=1$ giving:

$$
p^{*}=P_{o}(2 /[\gamma+1])^{\gamma / \gamma-1}
$$

The new pressure value, $p^{*}$, the sonic pressure at the throat, provides a path to another file of temperaturedependent information. Within the array of information, the initial entropy value is queried, $S_{o 1}$. The major assumption of isentropic flow means that $S^{*}=S_{0}$. The first iteration of the analysis is now complete because once $p^{*}$ and $S^{*}$ are known, the remaining sonic parameters at the throat are fixed and are taken from the CEA database. For example, the values of $T^{*}, h^{*}$, and the sonic velocity, $a^{*}$, can be taken from the data.

The iteration integrity is judged by the velocity of the gas, which can be calculated with energy balance shown in Eq. (9). The left side of Eq. (9) is the stagnation state with zero velocity $\left(u_{0}=0\right)$ and the right side of the equation represents conditions at the throat. Using this result and arranging and solving for sonic velocity leads to Eq. (10).

$$
\begin{aligned}
& h_{0}+u_{0}^{2} / 2=h_{1}+u_{1}^{2} / 2 \\
& u_{1}=a^{*}=\sqrt{2\left(h_{o}-h^{*}\right)}
\end{aligned}
$$


Table 1. Table of Thermodynamic properties. This table shows an array of data for a single pressure at varying temperature for a specific air-argon flow composition, generated with CEA.

THERMODYNAMIC PROPERTIES

$\begin{array}{lrrrrrrrrrrrrrr}\text { P, BAR } & 8.75 & 8.75 & 8.75 & 8.75 & 8.75 & 8.75 & 8.75 & 8.75 & 8.75 & 8.75 & 8.75 & 8.75 & 8.75 \\ \text { T, K } & 3500 & 3750 & 4000 & 4250 & 4500 & 4750 & 5000 & 5250 & 5500 & 5750 & 6000 & 6250 & 6500 \\ \text { RHO, KG/CU M } & 8.8539-1 & 8.1117-1 & 7.4390-1 & 6.8464-1 & 6.3400-1 & 5.9128-1 & 5.5486-1 & 5.2302-1 & 4.9434-1 & 4.6777-1 & 4.4255-1 & 4.1811-1 & 3.9411-1 \\ \text { h KJ/KG } & 4189.17 & 4830.96 & 5534.21 & 6245.22 & 6911.62 & 7513.73 & 8068.73 & 8612.79 & 9186.15 & 9828.28 & 10578.1 & 11474.7 & 12556.9 \\ \text { U, KJ/KG } & 3200.9 & 3752.27 & 4357.97 & 4967.18 & 5531.49 & 6033.88 & 6491.76 & 6939.82 & 7416.12 & 7957.71 & 8600.89 & 9382.01 & 10336.7 \\ \text { G, KJ/KG } & -26153.8 & -28343 & -30577.4 & -32856.2 & -35175.8 & -37530.7 & -39916 & -42328.6 & -44767.6 & -47234.1 & -49730.6 & -52261.4 & -54831.5 \\ \text { S, KJ/(KG)(K) } & 8.6694 & 8.8464 & 9.0279 & 9.2003 & 9.3528 & 9.483 & 9.5969 & 9.7031 & 9.8098 & 9.9239 & 10.0515 & 10.1978 & 10.3674 \\ \text { M, (1/n) } & 29.446 & 28.905 & 28.275 & 27.649 & 27.11 & 26.688 & 26.362 & 26.092 & 25.836 & 25.558 & 25.231 & 24.832 & 24.342 \\ \text { (dLV/dLP)t } & -1.01321 & -1.0197 & -1.02494 & -1.02682 & -1.02512 & -1.02164 & -1.01839 & -1.01647 & -1.01626 & -1.01784 & -1.02131 & -1.02673 & -1.03409 \\ \text { (dLV/dLT)p } & 1.2254 & 1.3109 & 1.3647 & 1.3643 & 1.3191 & 1.2618 & 1.2205 & 1.2068 & 1.2228 & 1.2681 & 1.3424 & 1.4453 & 1.5746 \\ \text { Cp, KJ/(KG)(K) } & 2.3967 & 2.7192 & 2.8689 & 2.7817 & 2.5361 & 2.2937 & 2.1714 & 2.2082 & 2.405 & 2.7579 & 3.2666 & 3.9325 & 4.7491 \\ \text { GAMMAs } & 1.1957 & 1.1934 & 1.199 & 1.2112 & 1.2274 & 1.2417 & 1.2468 & 1.2402 & 1.2252 & 1.2075 & 1.1912 & 1.178 & 1.1685 \\ \text { SON VEL,M/SEC } & 1087.1 & 1134.6 & 1187.6 & 1244.2 & 1301.5 & 1355.5 & 1402.2 & 1440.4 & 1472.6 & 1502.9 & 1534.7 & 1570.1 & 1610.7\end{array}$

This calculation for the sonic velocity is a check on whether or not the iterative procedure has converged. If the sonic velocity in the CEA table matches the calculated sonic velocity from Eq. (10), then one can be confident that the stagnation state conditions have been determined; the Mach number at the throat is indeed unity. Often this will not occur at the first iteration. When the velocity of the gas at the throat is not equal to the sonic velocity provided by CEA, the program returns to the stagnation state enthalpy prediction and adjusts it; corresponding parameters change as a result. The adjustment to the enthalpy is an average of the current stagnation and throat values. Although this is somewhat of a rough-and-ready way to improve the guessed value, it seems to provide solution convergence within a few iterations.

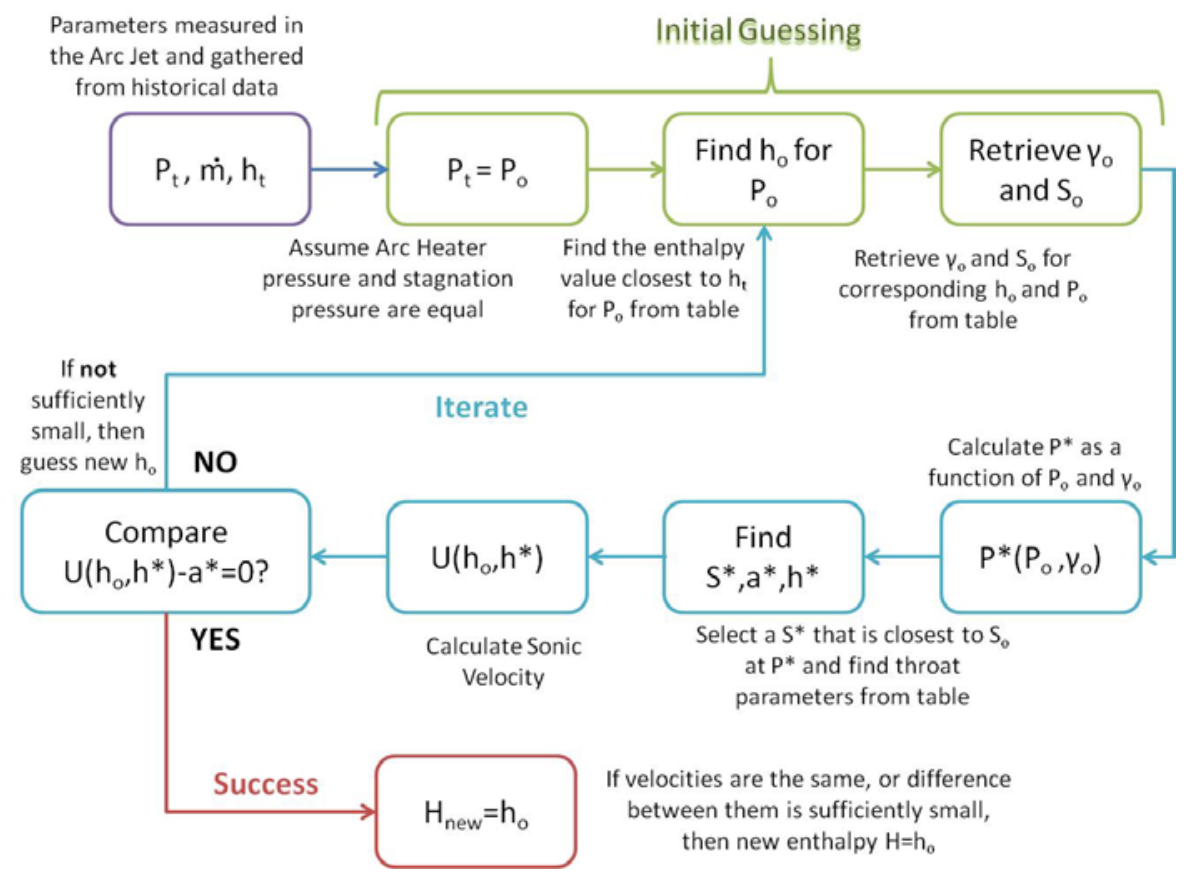

Figure 2: Flow diagram for iterative solution for enthalpy in Matlab.

\section{Results}

Flow rate and pressure values need to have been recorded in order to calculate a sonic flow parameter (per Eq. 3) and have the test be considered 'usable'. The database of AHF calorimeter tests contained 1240 usable tests; the database of IHF calorimeter tests contained 259 usable tests, by this criteria. Of the usable cases, a record was kept 
of which cases converged to matching sonic speed values over a nine-iteration allowance. Tests that did not converge were eliminated from the data set in further calculations.

The value of nine iterations was originally chosen as a guess and later examined for validity. With a nine iteration cap on the process, $65 \%$ of the IHF cases and $87 \%$ of the AHF cases converged. The percentage of convergence varied little when the limit was adjusted from 5 iterations through 20 iterations. For the AHF, there was one test case that had previously converged that failed to do so when the limit was reduced to 5 iterations, and no additional cases that converged when the limit was raised to 20 iterations. It was found that the average value of the constant $^{* *}, c$, being determined changed less than one percent when testing for an optimal convergence iteration limit.

A new constant, $c$, is calculated for each new value of bulk enthalpy. The calculation is simple because the exponent from Eq. (4) is set to 2 and the sonic flow parameter, $\sigma$, is a measured quantity. Once a new constant has been calculated for each test case, an average of the constants is used for the calculations and comparisons.

The constant, $c$, shows little to no dependence on the amount of argon in the flow. Figure (3) shows the calculated values of $c$ as a function of argon percentage in both the AHF and IHF arc jet flows, and while there is some deviation, it does not appear to depend on the amount of argon.
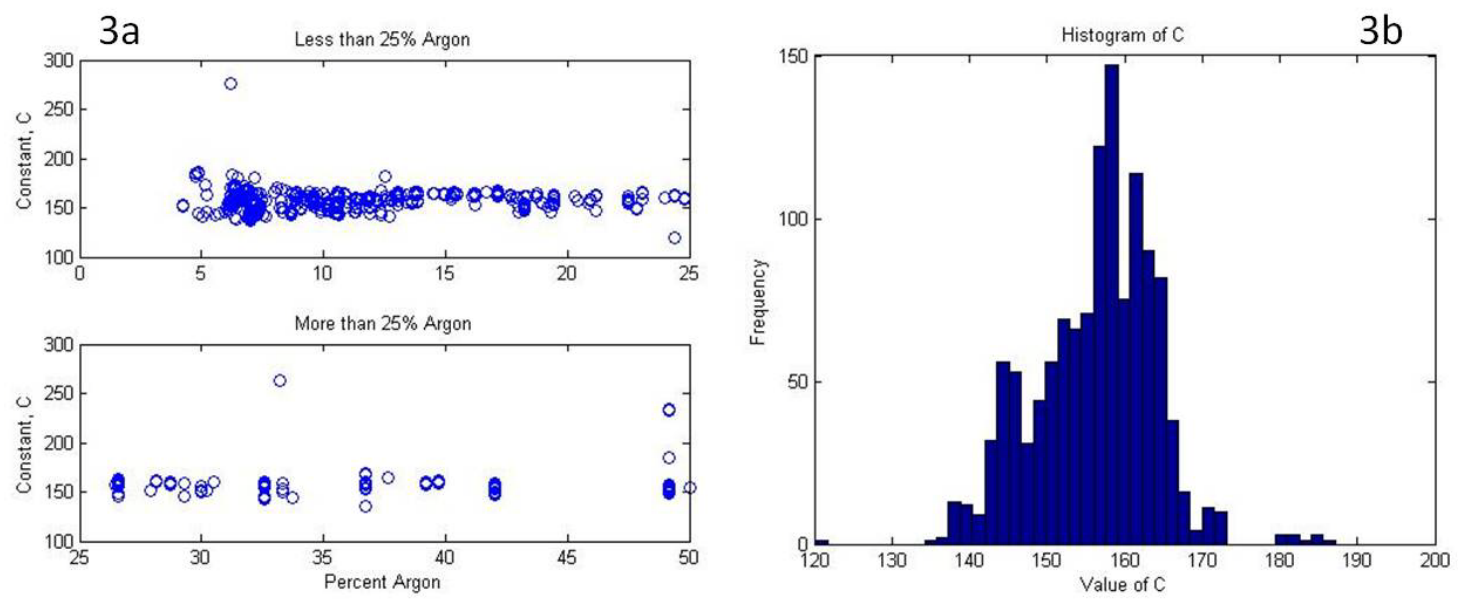

Figure 3: Variation of the calculated constant, $c$, with a) the quantity of argon present in the flow and b) histogram showing the frequency of values of $c$.

As a first pass, a coarse set of CEA data and the enthalpy calculated by the Winovich method were used for the initial guess in the iterative process described in Fig. (2). The resulting values for the constant, c, were the following: the AHF database yielded a constant of 142.6 averaged over 1240 cases; the constant for the 259 IHF cases was 121.3. A weighted average of the two constants (one from each facility) over all cases, provided a value of 138.9, which was less than the constant reported by Shepard et.al. and an increase over the constant presented by Winovich (after converting to SI units.)

Following the initial results, CEA was used to create the finer set of thermodynamic property data previously described in Section II. The finer data set results in a higher accuracy of the final enthalpy calculation but is also the cause of data attrition from non-convergence. A constant of 155.7 resulted when averaged over 1076 converging AHF cases. The constant for the 168 converging IHF cases was 162.2. A weighted average of the constants over all usable cases was 156.6, which is about a 1.5\% decrease from the Shepard value and about a $27 \%$ increase over the constant presented by Winovich. Results for the constant, $c$, were almost identical if the calculated Shepard value of bulk enthalpy was used as the initial enthalpy guess, with the advantage that more cases converged and the average value for the constant was 155.8 .

** The constant, c, referred to repeatedly in this work as the non-dimensional constant, is not actually without dimension. Physically the number has no dimensional significance, however a dimensional analysis results in that constant having units of $\mathrm{KPa} / \mathrm{atm}$. These units could have been massaged out of the constant easily enough but the resulting comparisons with previous work and the use of historical data would have been much more difficult. 
Equations (4) and (5) show the expressions for the Winovich and Shepard calculations. The new expression for bulk enthalpy calculation is shown here in Eq. (11), has units of MJ/kg, and is referred to as the "New" expression for clarity.

$$
H_{0}=(155.8 / \sigma)^{2}
$$

Some statistics are shown here in an attempt to address overall quality of fit of the different enthalpy calculation methods. These statistics are presented in Table (2) and are calculated with reference to the measured enthalpy by energy balance values for each data case used. A graphical fit of EB2 values and bulk enthalpy values calculated using all three methods are shown in Fig. (4). The hypothesis was that a new, dimensionally-correct, calculation of bulk enthalpy in arc jet flow would be in better agreement with the EB2 values obtained per the ASTM method. ${ }^{12}$ Figure 4 and Table 2 fail to validate this hypothesis by showing that all three methods are statistically equivalent at predicting EB2 values.

Table 2: Initial Statistics. Initial error calculations based on how the three methods compare with the measured EB2 values.

\begin{tabular}{|c|c|c|c|}
\hline \multirow{2}{*}{ Method } & \multirow{2}{*}{ Constant } & $\begin{array}{c}\text { Coef. Of Determination } \\
\left(\mathbf{R}^{2}\right)\end{array}$ & $\begin{array}{c}\text { Average \% } \\
\text { Error }\end{array}$ \\
\cline { 3 - 4 } & & \multicolumn{2}{|c|}{ With Respect to EB2 data } \\
\hline Shepard & 158.7 & 0.605 & 28.8 \\
\hline Winovich & 123 & 0.544 & 27.3 \\
\hline New & 155.8 & 0.606 & 28.4 \\
\hline
\end{tabular}

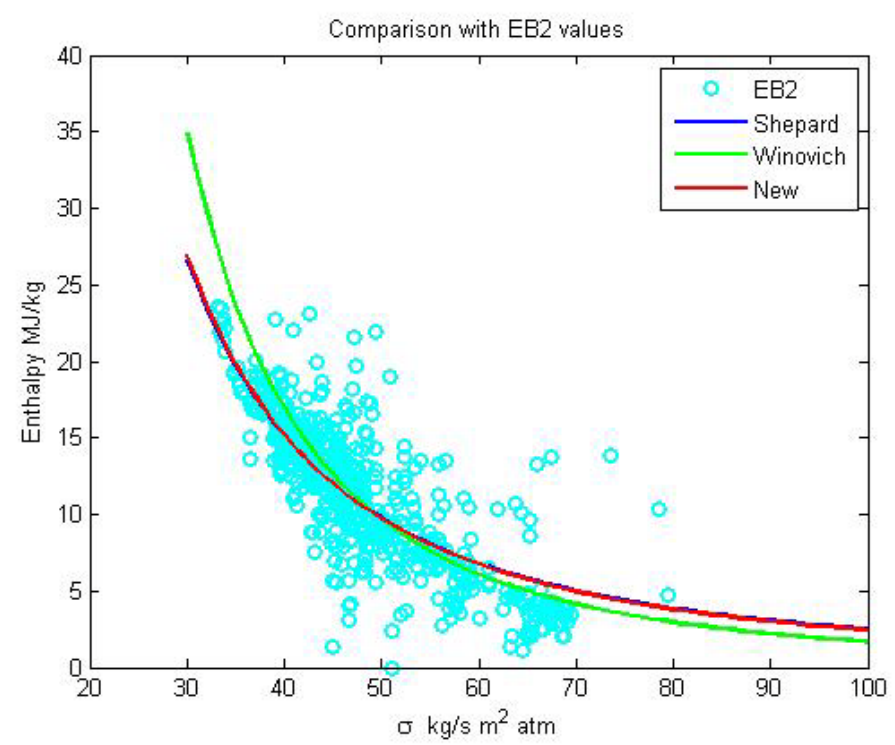

Figure 4. Enthalpy calculations. Comparison of the three enthalpy calculations with the measured EB2 values from both the IHF and AHF Facilities.

\section{Uncertainty analysis}

Uncertainty is calculated for each of the Enthalpy by Energy Balance measurements at the time that the data is compiled. A formal uncertainty analysis for the Winovich and Shepard bulk enthalpy calculations was performed along with the uncertainty analysis for the new method. This analysis is described in the following section. The goal of the uncertainty analysis is to show statistical similarity or dissimilarity between the different enthalpy values calculated and reported in the ARC arc jet facilities. 


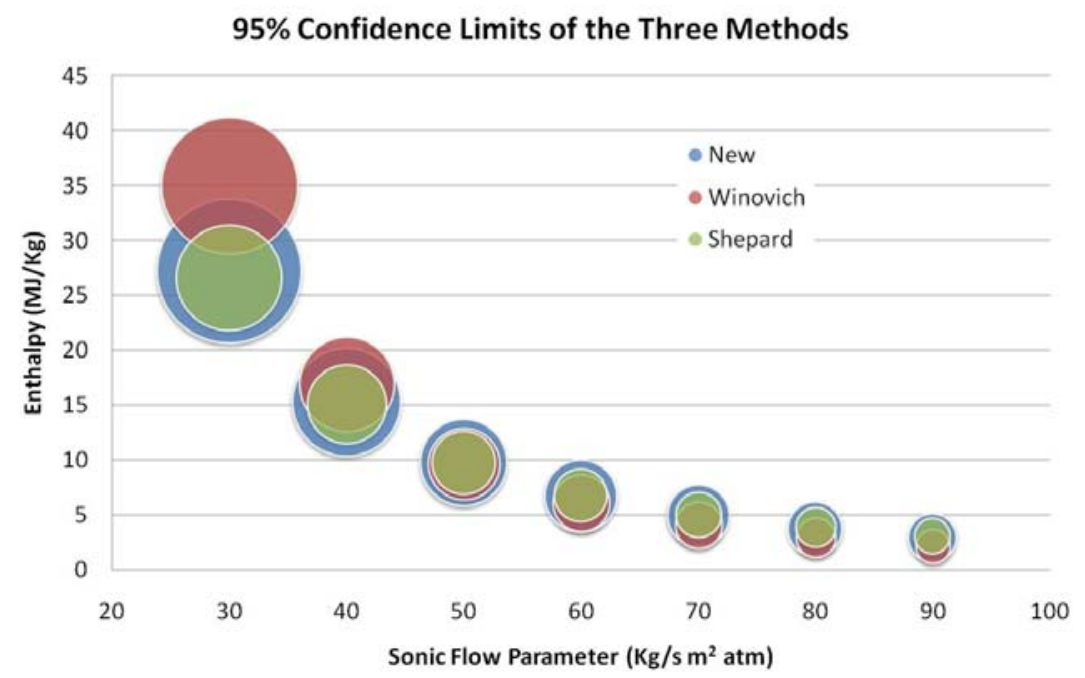

Figure 5: Two-sigma confidence limits shown for each of the three enthalpy calculations over a range of sonic flow parameters.

For a range of sonic flow parameters, the enthalpy and uncertainty have been calculated for the three enthalpy equations (Eqs 4, 5, and 11). Some assumptions were made including a five percent variability with 95\% confidence of the facility parameters of interest: mass flow rate of the test gases, arc heater pressure, and throat area. These assumptions are made for all three analyses so that any inaccuracy is equally shared in the results. In addition, the uncertainty of the constant used in both the Winovich and Shepard equations is unknown and was assumed to have five percent variability and $95 \%$ confidence. A notable advantage to the New method of enthalpy calculation is that we now put a known uncertainty on the value of $c$ because it has been calculated repeatedly. The new constant, $c$, value has a standard deviation of 9.5 about the mean.

Figure 5 is a bubble chart showing the results of the uncertainty analysis. The area of each bubble represents the 95\% confidence associated with the enthalpy calculation at the given sonic flow parameter. Each bubble is centered at the calculated value of enthalpy and the surrounding area can be thought of as the 2-sigma limits on the probability density graph for the calculation. The New method appears to have a larger confidence limit but this is most likely more accurate. Generic uncertainties were applied to the other methods that may have been artificially low.

\section{Discussion}

The iterative procedure outlined in the present paper requires an initial value (guess) for enthalpy. Using either the Winovich Sonic Flow Enthalpy (Eq. 4) or the Shepard enthalpy (Eq. 5) as the initial guess had very little impact on the final value of $c$; less than one percent change in the constant, $c$, was observed.

The data were further separated into three categories based on the degree of 'add air'. Add air is the secondary stream of room temperature air that can be added to the arc-heated stream to reduce enthalpy or adjust the heating condition. The three categories are: no add air, minimum add air (usually $55 \mathrm{~g} / \mathrm{s}$ ), and greater than minimum add air. Very little difference was seen in the calculated constant, $c$, as a function of add air. Figures $6 \mathrm{a}$ and $6 \mathrm{~b}$ show $c$ values plotted against the actual quantity of add air and the percentage of add air in the flow respectively.

The same logic was used to examine the data under different conditions of reported EB2 accuracy: less than 5\% uncertainty, $5 \%$ to $10 \%$ uncertainty, and greater than $10 \%$ uncertainty in the EB2 value. Once again little variation was observed in the constant that resulted from the calculations when separated into these categories, see Figure (7). Equation (11) was once again used for the examination of add air and EB2 accuracy. 

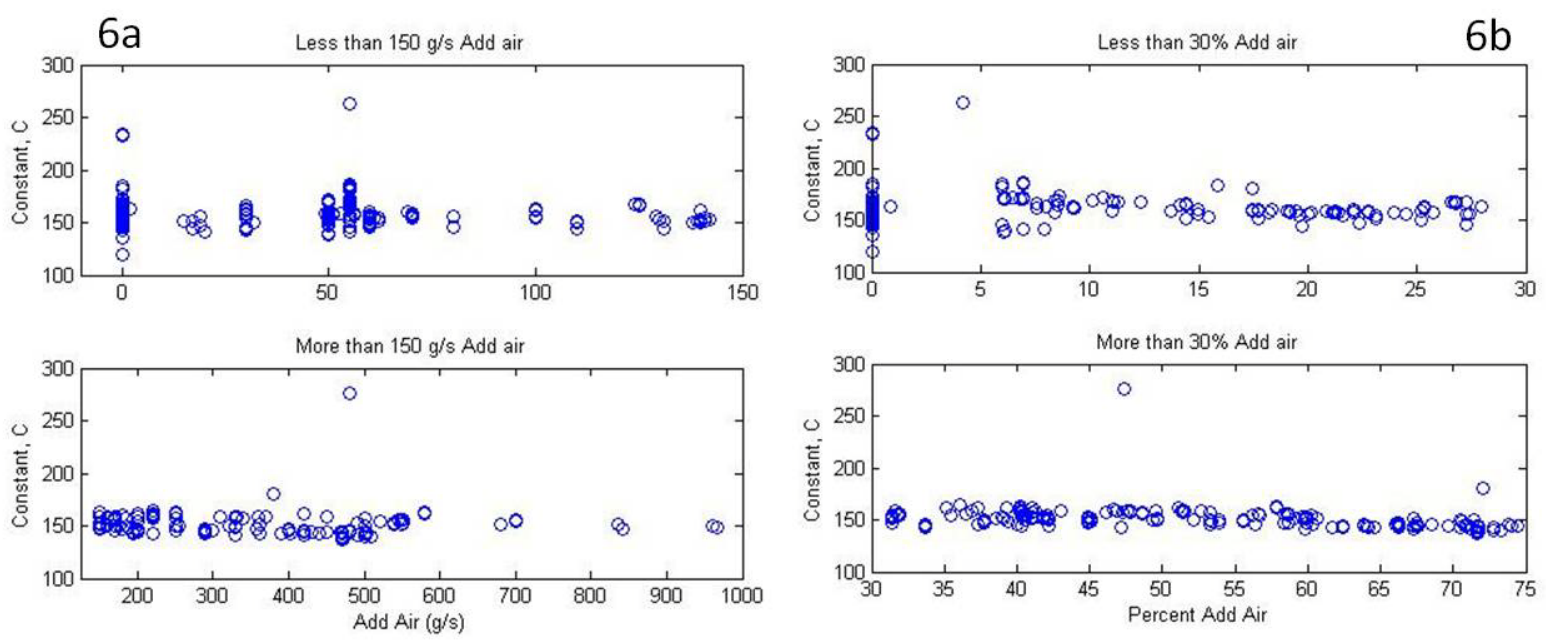

Figure 6: Variations in the constant, $c$, with a) quantities of add air and b) percentage of add air in the flow.
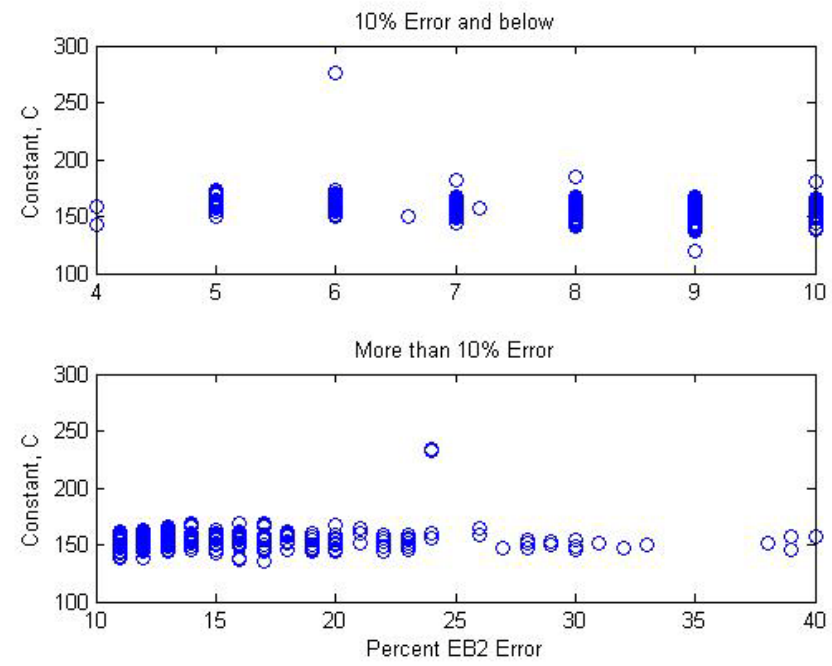

Figure 7: Variations in the constant, $c$, as a function of the error associated with the EB2 values.

The statistical similarity of the three methods seems apparent, especially at larger sonic flow parameters and lower enthalpy values. At enthalpy values less than $20 \mathrm{MJ} / \mathrm{kg}$, the three methods would produce the same results given the associated confidence limits. Recall that this is the range of enthalpies where the Winovich equation is considered to be valid.

The three methods compare with the measured EB2 values in much the same way, as is seen in Figure 4 and Table 2. From the figure, it appears that at low values of the sonic flow parameter the Shepard and New calculations are better calculations for enthalpy. However, a similar argument can be made in favor of the Winovich calculation at high values of the sonic flow parameter.

\section{Conclusions}

While the results of this work are qualitatively similar to those of Winovich and both qualitatively and quantitatively similar to Shepard's, the data set used in this analysis is more than 50 times larger than what was used by the previous investigators. The agreement shown here is a credit to those scientists' successful use of the small data sets that they had at their disposal and lends confidence to the results presented here. The new calculation has been shown to be equally, if not more, accurate than the Winovich and Shepard calculations when compared to EB2 
calculations. Possibly the most notable conclusion and advantage of this work is that the new calculation is dimensionally correct.

The uncertainty analysis in this study is a start to a much larger effort. Here, the large data set was used to determine confidence limits for a new constant, $c$. A more precise uncertainty analysis of these methods, and other arc jet calculations, could be performed when more precise error limits are associated with other arc jet parameters. The global assumption of five percent variability is conservative in some cases and perhaps generous in others.

The analysis presented herein is valid for isentropic flow. The results can be considered an upper-limit estimate for the bulk enthalpy when the flow deviates from the isentropic flow assumption. The data used in this study came from two facilities, each with a different nozzle throat area, and a variety of nozzle exit diameters, and over a wide range of facility operating conditions. The results derived are considered valid for the arc jets at NASA ARC. There has been debate over the years as to which method provides a better estimate of bulk enthalpy for the arc jet facilities at ARC. In the absence of EB2 data, or in light of questionable EB2 data, it is recommended that the new equation presented here, Eq. (11), be used to estimate the bulk enthalpy content of the flow.

\section{Acknowledgements}

This research was supported by the NASA-SCAP (Strategic Capabilities Assets Program) which provides critical financial support of the arc jet operational capability at NASA Ames Research Center.

Sandia National Laboratories is a multi-program laboratory managed and operated by Sandia Corporation, a wholly owned subsidiary of Lockheed Martin Corporation, for the U.S. Department of Energy's National Nuclear Security Administration under contract DE-AC04-94AL85000.

Many thanks to the contributors at NASA Ames Research Center for both input and continued support, specifically the Thermophysics Facilities and Aerothermodynamics Branches. Thanks also to Sandia National Laboratories center 2600 management for support and encouragement of this work.

\section{References}

\footnotetext{
${ }^{1}$ Prabhu, D., Saunders, D., Oishi, T., Skokova, K., Santos, J., Fu, J., Terrazas-Salinas, I., Carballo, E., Driver, D., “CFD Analysis Framework for Arc-Heated FlowFields, I: Stagnation Testing in Arc-jets at NASA ARC”, AIAA-2009-4080, AIAA 41 ${ }^{\text {st }}$ Thermophysics Conference, San Antonio, TX., 2009.

${ }^{2}$ Hightower, T.M., Balboni, J.A., MacDonald, C.L., Anderson, K.F., and Martinez, E.R., "Enthalpy by Energy Balance for Aerodynamic Heting Facility at NASA Ames Research Center Arc Jet Complex”, $48^{\text {th }}$ International Instrumentation Symposium, The Instrumentation Systems and Automation Society, Research Triangle Park, NC, May 2002.

${ }^{3}$ Terrazas-Salinas, I., Cornelison, C., “Test Planning Guide for ASF Facilities”, NASA Ames Research Center, A029-9701XM3 Rev. C, April 2009.

${ }^{4}$ Winovich, W., "On the Equilibrium Sonic-Flow Method for Evaluating Electric-Arc Air-Heater Performance”, NASA TN D-2132, 1964.

5 Shepard, C., Milos, F., Taunk, J., “A Sonic Flow Equation for Electric Arc Jets”, AIAA-93-3183, AIAA 24 Plasmadynamics \& Lasers Conference, Orlando, FL, 1993.

${ }^{6}$ Vincenti, W.G., Kruger, C.H., Introduction to Physical Gas Dynamics, Krieger Publishing Company, Malabar, FL 1965.

${ }^{7}$ Yoshikawa, K. K., Katzen, E. D., "Charts for Air-Flow Properties in Equilibrium and Frozen Flows in Hypervelocity Nozzle”, NASA TN D-693, 1961.

${ }^{8}$ V. Watson and E. Pegot, "Numerical Calculations for the Characteristics of a Gas Flowing Axially Through a Constricted Arc”, NASA TN D-693 (1961)

${ }^{9}$ Gordon, S., McBride, B. J., “Computer Program for Calculation of Complex chemical Equilibrium Compositions and Applications”, NASA Reference Publication 1311, 1994.

${ }^{10}$ MATLAB R2008a, The MathWorks Inc., Version 7.6.0.324, February 10 2008, copyright 1984-2008

${ }^{11}$ Mark Hightower, personal communication, April 2009

12 “Standard Practice for Measuring Plasma Arc Gas Enthalpy by Energy Balance”, ASTM Standard Designation: E341-96, American Society for Testing and Materials, West Conshohocken, Pa., 1996.
} 